BMJ Paediatrics Open

\section{Pharmacokinetics of prednisolone in children: an open-label, randomised, two-treatment cross-over trial investigating the bioequivalence of different prednisolone formulations in children with airway disease}

To cite: Haslund-Krog SS, Schmidt M, Mathot R, et al. Pharmacokinetics of prednisolone in children: an open-label, randomised, two-treatment cross-over trial investigating the bioequivalence of different prednisolone formulations in children with airway disease. BMJ Paediatrics Open 2019;3:e000520. doi:10.1136/ bmjpo-2019-000520

Received 14 May 2019 Revised 3 September 2019 Accepted 8 September 2019

Check for updates

(c) Author(s) (or their employer(s)) 2019. Re-use permitted under CC BY-NC. No commercial re-use. See rights and permissions. Published by BMJ.

For numbered affiliations see end of article.

Correspondence to Dr Sissel Sundell Haslund-Krog; sissel.sundell.haslund-krog.01@ regionh.dk

\section{ABSTRACT}

Introduction One in three Danish children under 3 years of age experience asthma-like symptoms, and one-third will later be diagnosed with asthma. Oral prednisolone is used in various formulations to treat acute asthma. However, the potential differences in bioequivalence between these formulations have never been examined in children despite interchangeable use in clinical practice.

Methods and analysis An open-label, randomised, two-treatment cross-over trial investigating the bioequivalence of different prednisolone formulations in children with airway disease.

The included patients ( 6 months -11 years of age) are admitted to the Department of Paediatric and Adolescent Medicine Nordsjællands University Hospital, Hillerød, with asthma or asthma-like symptoms.

The primary objective is to assess the bioequivalence between different prednisolone formulations herein area under the concentration time curve, Cmax and Tmax using saliva samples. The secondary objectives are to evaluate tolerability (five-point face scale), adverse events and severity of the disease. If the patient has an intravenous access for other purposes, the saliva samples will be validated with plasma samples.

A total of 66 evaluable patients are needed according to European Medicines Agency Guideline on bioequivalence. Ethics and dissemination Traditional pharmacokinetic trials are burdensome due to the extent of blood samples necessary to capture the time-dependant drug profile. Saliva sampling is far more acceptable for paediatric patients. In addition, this trial adheres to standard dosing strategies. No additional venepunctures are performed, and no additional prednisolone doses are administered. Guidelines for paediatric bioequivalence trials are warranted.

Trial registration number The Danish Medicines Agency EudraCT: 2017-003590-33, The Ethics Committee case no: $\mathrm{H}-17027252$, and the Danish Data Protection Agency: BFH-2017-103, I-Suite no.: 05935.

\section{What is already known on this topic?}

- Various prednisolone formulations are frequently used to treat asthma-like symptoms in children; the potential differences in bioequivalence between these formulations have never been examined.

- Pharmacokinetic studies have traditionally been burdensome for the paediatric population due to numerous blood samples.

- It is important to study the acceptability of paediatric formulations, since manipulating medicine or introducing different formulations may diminish or enhance tolerability.

\section{What this study hopes to add?}

Paediatric bioequivalence data on prednisolone ora solution, crushed tablets and orodispersible tablets compared with whole tablets.

- Prednisolone concentration data are collected using saliva samples, validated with plasma samples if the patient has an intravenous access for clinical purposes.

- Acceptability data on different prednisolone formulations using a modified Wong-Baker face scale.

\section{INTRODUCTION}

One in three Danish children under 3 years of age experience asthma-like symptoms, and one-third will later be diagnosed with asthma. ${ }^{1}$ Therapeutic management of acute asthma-like symptoms in infants and children has been repeatedly discussed. ${ }^{2}$ Oral glucocorticoids are frequently added to inhaled beta-2-agonist if the patient does not respond to the initial treatment. However, no therapeutic interval exists for glucocorticoids, and 
no clinical biomarker has been deveopled to asses efficacy. ${ }^{3}$

Furthermore, in Denmark, only intravenous formulations and prednisolone or hydrocortisone tablets are licenced for patients $<18$ years of age. ${ }^{4}$ Infants and children who are unable or unwilling to swallow a tablet or where dosing is based on body weight are urged to use either an extemporaneous oral liquid formulation, crushed or split tablets. Splitting tablets may lead to dose inaccuracy, and in one study, crushing hydrocortisone tablets lead to therapeutic failure. ${ }^{5}$ Extemporaneous formulations are not subject to randomised controlled trials as licenced formulations and bioequivalence (BE) are not determined between different formulations with the same active ingredience. ${ }^{56}$ Moreover, the paediatric population is a heterogeneous group of patients ranging from premature neonates to adolescents. The wide physical and developmental differences influence both pharmacokinetic (PK) and pharmacodynamic parameters. These issues are generally poorly studied. ${ }^{7}$

BE studies compare both the rate (the time to Cmax$=$ Tmax $)$ and extent of absorption (area under the concentration time curve (AUC) of various drug formulations. Regulatory guidelines on BE state that two treatments are not different from one another if the $90 \%$ CI of the ratio of a log-transformed exposure measure (AUC and/or Cmax) falls within the range of $80 \%-125 \% .^{8}$

To date, one BE study with prednisolone have been performed in healthy adults. ${ }^{9}$ Francisco et at ${ }^{9}$ examined AUC, Tmax and Cmax of five commercially oral prednisone tablets compared with one reference solution in 18 healthy adults. ${ }^{9}$ No statistically significant differences were found between the tablets. Non-significant variations were seen between the solution and tablets except for Tmax, which was 0.53 and $1.2-1.7$ hours, respectively. ${ }^{9}$ A bioavailability study from Georgitis $e t a l^{10}$ compared liquid prednisone, prednisone tablet and intravenous prednisolone ${ }^{10}$ and found that the AUC for liquid and tablet were significantly different. ${ }^{10}$ No studies have been performed in a target patient population, taking into consideration factors such as comorbidities, comedication and physiological factors including first pass metabolism, gastric $\mathrm{pH}$ and bacterial flora, which are highly relevant in infants and children.

However, traditional PK trials are burdensome due to the extent of blood samples, necessary to capture the time-dependant drug profile. New non-invasive methods such as saliva sampling are far more acceptable for paediatric patients. In a recent adult population PK study, free prednisolone concentrations correlated well with salivary concentrations. ${ }^{11}$ The same study set-up were later applied in a population PK model in paediatric patients $(\mathrm{n}=104)$ with nephrotic syndrome. ${ }^{12}$ None of the studies included more than one formulation.

The importance to study the acceptability of paediatric formulations has recently been endorsed since manipulating medicine or introducing different formulations may diminish or enhance tolerability. ${ }^{13}$ Acceptability of medicines influence adherence to therapeutic regimens and outcomes. No validated method to access acceptability of paediatric medicine exists, and many different scales have been used most commonly hedonic face scales or visual analogue scale (VAS) ${ }^{13}$ Lucas-Bouwman et $a l^{14}$ investigated the acceptability of prednisolone oral solution and crushed tablets using VAS taste scale. The oral solution obtained the best score and no vomiting was observed in the oral solution treatment group compared with $23 \%$ in the other group. Aljebab et al $l^{15}$ showed that dexamethasone elixir was more palatable than prednisolone tablets, soluble tablets and syrup using af five-point hedonic face scale in children below 12 years of age in the UK and Saudi Arabia. ${ }^{15}$ Prednisolone syrup and soluble tablets caused less vomiting than tablets. ${ }^{15}$

\section{OBJECTIVES}

We aim to investigate the bioeqvalence of different prednisolone formulations in three different age-groups and secondary to investigate the tolerability, adverse events, severity of the disease and validation of saliva samples, table 1 .

\section{METHODS AND ANALYSIS}

A single-centre, randomised, open-label two-treatment cross-over design.

Children with asthma or asthma-like symptoms admitted to the Department of Paediatric and Adolescent Medicine, Nordsjællands University Hospital, Hillerød, are eligible for inclusion.

\section{Population}

Eligibility criteria are described in table 2. Patients are stratified by age in accordance with European Medicines Agency (EMA) guidelines into three groups, that is, 6-23 months, 2-5years and 6-11 years. ${ }^{16}$ Two different formulations of prednisolone as $1 \mathrm{mg} / \mathrm{kg} /$ day will be administered to each participant for two consecutive days (figure 1). A control group of school children age 6-11 years will receive whole tablets. In total, four different formulations will be investigated (see figure 1). All trial medicine is labelled, packed and distributed by the Regional Pharmacy in accordance with GMP Annex $13,{ }^{17}$ except the high-strength oral prednisolone solution $20 \mathrm{mg} / \mathrm{mL}$, which is 'ward medicine' and does not require special labelling. A separate accountancy is kept for each formulation of prednisolone.

\section{Patient involvement}

Patients were not directly involved in the design of this study.

\section{Saliva samples}

The primary and secondary outcomes are assessed at predefined time points shown in table 3 and figure 1 . Patients are randomised to one of two sampling groups, A and B, figure 1. Sampling times are calculated on 
Table 1 Primary and secondary objectives and outcomes in the POP child trial

\section{Objectives and outcomes}

\section{Primary objective}

To investigate the bioequivalence of different prednisolone formulations, that is, crushed tablets, oral solution and orodipersible tablets to characterise their bioequivalence compared with standard whole tablets (controls). Herein compare the AUC, Cmax and Tmax.

\section{Secondary objective}

1. Acceptability of different formulations assessed by patient preferences and number of attempts

2. Adverse events.

3. Modified Pulmonary Index Score (MPIS) and Paediatric Early Warning Score (PEWS).

4. Validation of the saliva samples using plasma samples.

\section{Primary outcome}

Concentration time data on prednisolone concentration measured in saliva to determine AUC, Tmax and Cmax.

\section{Secondary outcome}

1. Acceptability of different formulations measured by a modified Wong-Baker face scale (see figure 2).

2. Adverse events registration.

3. MPIS and PEWS score at administration time for doses 1 and 2.

4. Concentration-time data on prednisolone concentration measured in plasma to determine AUC, Tmax and Cmax

AUC, area under the concentration time curve.

the basis of a previous study. ${ }^{12}$ Each child will follow the same time schedule for both doses. The samples will be collected using the SalivaBio Children's Swab and the SalivaBio Oral Swab (exclusively from Salimetrics, State College, Pennsylvania, USA), synthetic swabs specifically designed to improve volume collection and increase participant compliance. Saliva will be removed from the swabs by centrifuge $(15 \mathrm{~min}$ at $2500 \mathrm{rpm})$ and stored in cryovials at $-20{ }^{\circ} \mathrm{C}$ for maximum 2 weeks and hereafter at $-80^{\circ} \mathrm{C}$ until analysis. Concentrations of prednisolone and its inactive metabolite prednisone will be determined by liquid chromatography with mass

Table 2 Inclusion and exclusion criteria for the POP child trial

Inclusion and exclusion criteria for the POP child trial

Inclusion criteria Exclusion criteria

Children from 6 months to 11 years of age, both gender, all ethnicities.

Unable to comply with trial procedures, for example, inability to swallow tablets, not cooperative for saliva sampling and so on.

\begin{tabular}{|c|c|}
\hline $\begin{array}{l}\text { Asthma-like symptoms or } \\
\text { asthma. } \\
\text { Admitted to the paediatric } \\
\text { or emergency department. }\end{array}$ & $\begin{array}{l}\text { Hypersensitivity to the active } \\
\text { substance prednisolone. }\end{array}$ \\
\hline $\begin{array}{l}\text { Intended prednisolone } \\
\text { treatment. }\end{array}$ & $\begin{array}{l}\text { Menarche or testis volume } \\
>4 \mathrm{~mL} \text { (evaluated in children } \\
\text { above } 7 \text { years of age). }\end{array}$ \\
\hline $\begin{array}{l}\text { Informed written consent } \\
\text { from both parents or legal } \\
\text { guardian. }\end{array}$ & \\
\hline
\end{tabular}

\section{Withdrawal criteria:}

\section{Non-compliance with the}

given formulations after

three attempts.

POP, Pharmacokinetics of prednisolone. spectrometric detection. The assay is validated according to the EMA guideline 'Guideline on bioanalytical method validation' (EMEA/CHMP/EWP/192217/2009 Rev. 1 Corr. $2^{* *}$ Committee for Medicinal Products for Human Use). Analyses are performed in accordance with ISO 15189. Concentrations will be determined in $10 \mu \mathrm{L}$ saliva or plasma. In the latter case, both total and unbound concentrations will be determined. The lower limit of quantification is $2 \mathrm{ng} / \mathrm{mL}$ for both compounds. Saliva samples are non-invasive samples and are considered a painless low-risk procedure. ${ }^{16}$ Saliva sampling of prednisolone and subsequent analysis has recently been performed in children with median 4.6 years of age using Salivette equipment. ${ }^{12}$ As this trial includes children from 6 months of age, we chose the Salimetrics equipment. If any child has a cubital intravenous access at inclusion, additional blood samples will be taken time equivalent to the saliva samples. In order to assess plasma prednisolone for validation of the saliva samples. No additional venepunctures will be made.

\section{Sample size}

The number of subjects required for a BE study depends on the expected deviation between formulations. ${ }^{18}$ Because of limited data on prednisolone PKs and BE of different formulations, sample size calculation is not feasible. In accordance with the EMA guideline on the investigation of $\mathrm{BE},{ }^{8}$ the number of subjects to be included in a study should preferably be based on sample size calculations and not be less than 12. To account for drop-outs, 14 patients were allocated to each formulation in the three different age groups and the control group (a total of 77 patients) (figure 1). The optimal number of participants is 66 evaluable patients.

\section{Randomisation}

The participants are randomised in the REDCap software (www.project-redcap.org). The REDCap software is mandatory to use in investigator-initiated research in 
\begin{tabular}{ll} 
Dose $1 \quad$ Dose 2 \\
\hline
\end{tabular}

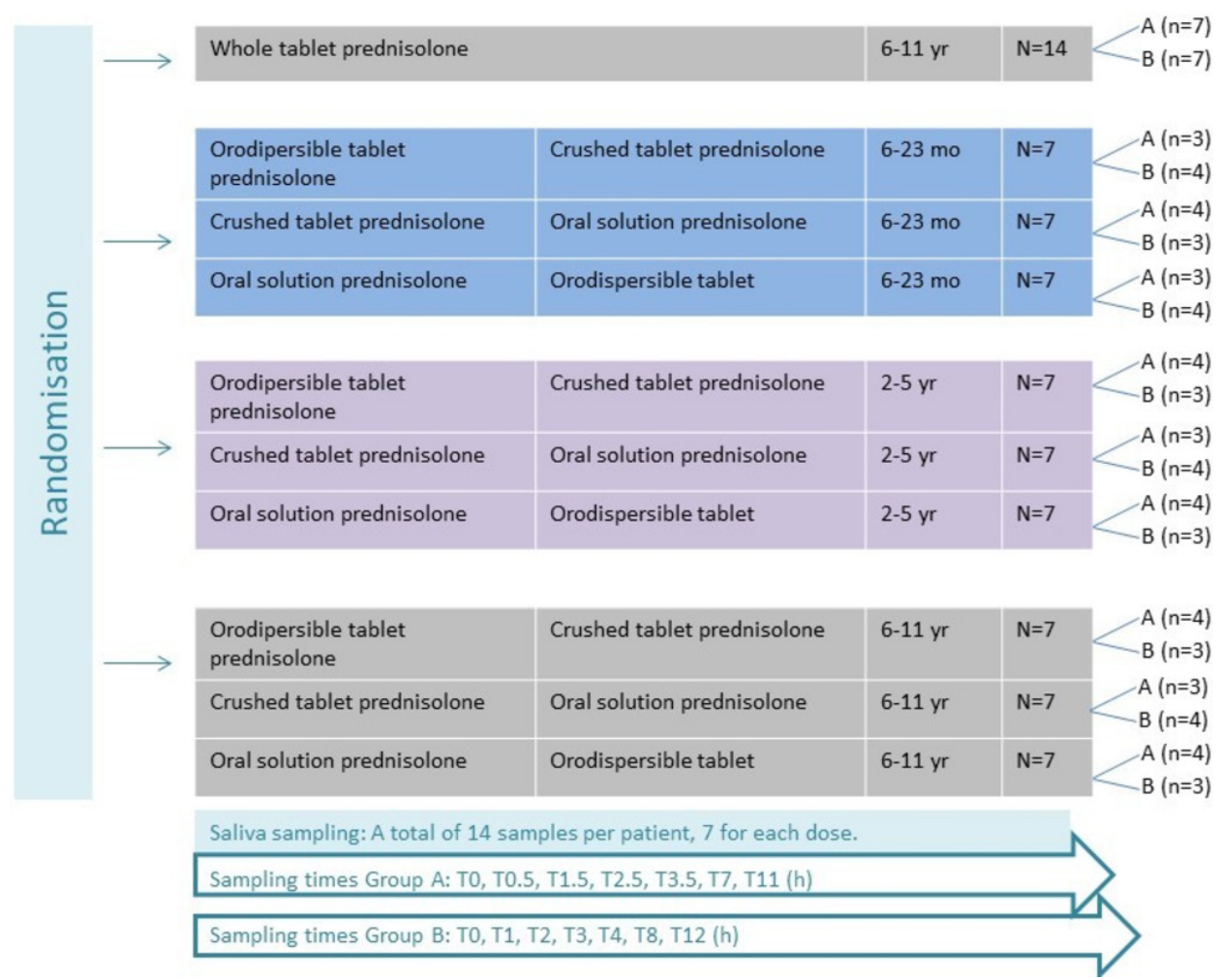

Figure 1 In total, four investigational medical products (IMPs) are used: 'Prednisolon DAK' whole tablets, 'Prednisolon DAK' crushed tablets (5 or $25 \mathrm{mg}$ ), extemporaneous prednisolone oral solution and SOLUPRED orodispersible tablets (5 or $20 \mathrm{mg}$ imported from France). The oral solution exists in two strengths, that is, $5 \mathrm{mg} / \mathrm{mL}$ (for children below $10 \mathrm{~kg}$ ) and $20 \mathrm{mg} / \mathrm{mL}$ (for children above $10 \mathrm{~kg}$ ).

the Capital Region in Denmark. The randomisation list is uploaded in REDCap and is blinded to everyone except a third person, who generates the list and has no other role in the trial. The participants are age stratified and will be block randomised to ensure an equal amount in each subgroup (figure 1 ). The child will receive a REDCap generated ID number including a combination of letters describing the formulations to be given. Administration of prednisolone is done open-label by the project staff or paediatric nurses.

\section{Statistical methods}

The AUC(0t) and Cmax parameters will be calculated using single subject compartmental modelling. Actual time of sampling will be used in the estimation of the PK parameters. The assessment of $\mathrm{BE}$ will be based on $90 \%$ CIs for the ratio of the population geometric means (test/reference), with the null hypothesis of $\mathrm{BE}$ at the $5 \%$ significance level (acceptance interval of $80.00 \%-$ $125.00 \%)^{8}$ using ANOVA or t-test. The comparison between the different age groups will be done in a stepwise manner, initially comparing the various subpopulation, for example, the 6-23 months old. If these data are comparable, we will pool the data and compare with the older age groups. The oldest age group will only then serve as control group across age population. If the data do not show BE, the bioavailability data will be reported separately for each age group.

The person responsible for the statistical analysis of saliva samples will be blinded to the formulations given. Due to the limited wash-out period, a test for carry-over will be addressed by examination of the pretreatment plasma concentrations in period 2 .

All patients who fulfils the eligibility criteria and have had at least one saliva sample taken will be analysed for demographic and baseline data. In the PK modelling, all saliva samples will be included. If any predose concentration is more than $5 \%$ of Cmax, this will be reported and handled appropriately in the final analysis.

\section{Ethics and dissemination}

Before prednisolone treatment is initiated, a patient information sheet is given to eligible patients and the trial is explained to their parents. Parents will have a minimum of $15 \mathrm{~min}$ to consider the consent, because treatment should not be unnecessarily delayed. Informed consent will be granted by parents or legal guardian, as all children are below 15 years of age. If one of the parents is not present, a written authority can be used. The consent process will be conducted by an investigator with training and experience with minors. The collected material is considered a research biobank. 
Table 3 The table illustrates the different trial activities.

\begin{tabular}{|c|c|c|c|c|c|}
\hline Visit & & Baseline & 1. dose & 2. dose & access \\
\hline Timeframe & Screening & $\begin{array}{l}\text { Before } \\
\text { treatment } \\
\text { start }\end{array}$ & $\begin{array}{l}\text { See individualised } \\
\text { timing of saliva } \\
\text { samples }\end{array}$ & $\begin{array}{l}\text { See individualised } \\
\text { timing of saliva } \\
\text { samples }\end{array}$ & $\begin{array}{l}\text { Same sampling times } \\
\text { as the saliva samples }\end{array}$ \\
\hline Eligibility & & & & & \\
\hline Informed consent & & $x$ & & & \\
\hline Inclusion and exclusion criteria & $\mathrm{x}$ & $x$ & & & \\
\hline Information to parents & $x$ & $\mathrm{x}$ & & & \\
\hline Other measurements & & & & & \\
\hline Other medicine & & $X^{*}$ & $X^{*}$ & $x$ & \\
\hline MPIS/PEWS & & $X^{*}$ & $X^{*}$ & $x$ & \\
\hline Assessment of adverse events & & $x$ & $x$ & $x$ & \\
\hline Weight & & $X^{*}$ & $X^{*}$ & $X^{*}$ & \\
\hline Effect parameters & & & & & \\
\hline $\begin{array}{l}\text { Prednisolone concentrations } \\
\text { measured in saliva samples }\end{array}$ & & $x$ & $x$ & $x$ & $x \dagger$ \\
\hline Secondary parameters & & & & & \\
\hline Tolerability & & & $\mathrm{x}$ & $x$ & \\
\hline
\end{tabular}

*Will share the same value or registration.

†Blood samples in addition to saliva samples.

MPIS, Modified Pulmonary Index Score; PEWS, Paediatric Early Warning Score.

\section{Safety}

Despite a large therapeutic index, it is important that all glucocorticoids are administered in the lowest effective dose as possible, since a considerable number of dose-dependent adverse drugs reactions (ADRs) are well described. ${ }^{4}$ A recent review found that the most frequent ADRs associated with short course oral corticosteroids (<14 days) in children were vomiting, changes in behavoiur and sleep disturbances with incidence rates of $5.4 \%, 4.7 \%$ and $4.3 \%$, respectively. Increased susceptability to infection were one of the serious ADRs ocurring in up to $1 \%$ of the children. ${ }^{19}$

\section{Trial limitations}

Alterations have been made in this trial compared with a classic BE trial to adapt to the relevant population. There are no existing guidelines for paediatric $\mathrm{BE}$ trials. A guideline is warranted since paediatric $\mathrm{BE}$ trials must be pragmatic in order to adapt to the paediatric population and to be conducted ethically.

Optimally, a randomised, two-period/three-period, two-sequence/three-sequence single-dose cross-over design would be used for healthy adults. This trial is a single-dose study performed during admission, limiting the possible number of doses/formulations per patient, with a theoretical washout period of 24 hours, as prednisolone is dosed once daily. The half-life of prednisolone is $2.5 \pm 0.5$ hours, ${ }^{20} 21$ and 24 hours should be enough time to ensure drug concentrations below quantification. However, as a precaution, predose samples are collected if prednisolone has been administered the day before inclusion. The saliva samples will be measured in 11 or 12 hours, respectively, which will be within five half-lives for many patients. Late night samples will be more difficult to collect if the participants are asleep and some might not be collected due to patient and parent preferences.

The reference product in this trial is whole tablets, since intravenous glucocorticoids are mainly used for severe asthma. The effect of fasting and fed are not within the scope of this trial since no effect on bioavailability was found in prednisolone whole tablets. ${ }^{4}$ To avoid prednisolone leftovers in the mouth, all children will be given water after administration of trial medicine. There can be differences in which the hedonic face scale is presented to the child, and this can be of some limitation (figure 2). Only 2-5 persons will include participants in this trial, minimising the variability.

We expect to have some dropouts due to: (1) non-compliance with the formulations and (2) due to earlier than expected discharge. In the control group with whole tablets data suggest that about $91 \%$ of 6 -year-old children can swallow a $7 \mathrm{~mm}$ tablet. ${ }^{13}$ The whole tablets are $8 \mathrm{~mm}(5 \mathrm{mg})$ and $10 \mathrm{~mm}(25 \mathrm{mg})$ but with break lines. We chose to include two strengths of prednisolone oral solution, to make sure the volumes were measurable and acceptable. ${ }^{13}$ Dropouts and inclusion 


\section{How is the medicine accepted? (A-E): (accessed by the child $\geq 6$ years of age or parent $<6$ years of age)}

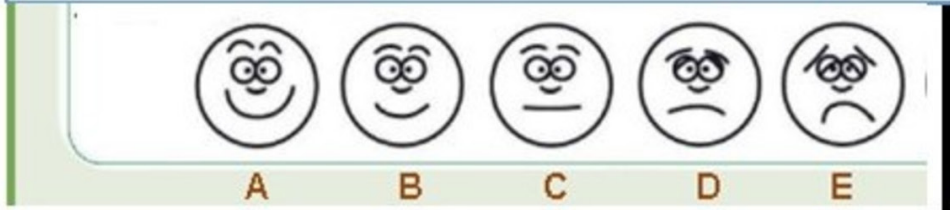

A
B

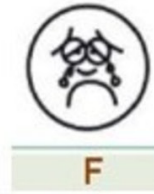

A Tastes good.

B Tastes a little less good, but is accepted.

C Tastes neither good nor bad

D Tastes bad, swallows with some difficulty

\section{$\mathbf{F}$ is evaluated separately and is only marked if the child vomits}

E Tastes really bad.

Figure 2 Modified Wong-Baker face scale to access tolerability of trial medicine. Self-reported tolerability of the medicine is collected from children older than 6 years of age. The options A-E is read aloud neutrally to the child. For children below 6 years of age, a parent is asked to evaluate the tolerability. Vomiting is registered separately but is included in the face scale as $\mathrm{F}$.

only during daytime could give some recruitment bias; however, as this is primarily a PK trial, we do not expect this to influence the results. This trial is conducted at one site which can limit the generalisability but will enhance the consistency.

\section{Trial registration}

This trial is an investigator-initiated trial from the Department of Clinical Pharmacology, Bispebjerg Hospital and the Department of Paediatric and Adolescent Medicine, Nordsjællands Hospital, Hillerød. The trial is approved by the Danish Medicines Agency EudraCT: 2017-003590-33, the Ethics Committee case no: H-17027252 and the Danish Data Protection Agency: BFH-2017-103, I-Suite no.: 05935. The trial is monitored by the GCP-unit Copenhagen University.

\section{Author affiliations}

'Department of Clinical Pharmacology, Bispebjerg Hospital, Copenhagen, Denmark 2Department of Paediatric and Adolescent Medicine, Hillerød Hospital, Hillerød, Denmark

${ }^{3}$ Department of Hospital Pharmacy - Clinical Pharmacology Unit, Amsterdam University Medical Centres, Amsterdam, The Netherlands

${ }^{4}$ Department of Biostatistics, Institute of Public Health, University of Copenhagen, Copenhagen, Denmark

${ }^{5}$ Department of Clinical Research, Hillerød Hospital, Hillerød, Denmark

${ }^{6}$ Institute for Clinical Medicine, University of Copenhagen, Copenhagen, Denmark

Contributors HH, SSH-K and IMJ were involved in the conception and design of the study. HH, SSH-K and IMJ wrote the protocol and applied for all permissions and funding. All authors were involved in preparing the study sites, acquisition of data and analysis, in writing the protocol article and in its revision prior to submission.

Funding The salary to the $\mathrm{PhD}$ student is covered by Department of Clinical Pharmacology and the Danish Regions. Costs for trial medicine, project staff and analysis of saliva samples are covered by the A.P. Møller Foundation, Captain Lieutenant Harald Jensen and Wife Foundation, The Danish Medical Research Grant and Aase and Ejnar Danielsens Foundation.

Competing interests None declared.

Patient consent for publication Not required.

Provenance and peer review Not commissioned; externally peer reviewed.

Data availability statement The article reports a protocol for an ongoing trial. Therefore, no trial data are available yet. All data relevant to the study are included in the article or can be proqured from the authors upon requst.
Open access This is an open access article distributed in accordance with the Creative Commons Attribution Non Commercial (CC BY-NC 4.0) license, which permits others to distribute, remix, adapt, build upon this work non-commercially, and license their derivative works on different terms, provided the original work is properly cited, appropriate credit is given, any changes made indicated, and the use is non-commercial. See: http://creativecommons.org/licenses/by-nc/4.0/.

\section{REFERENCES}

1 Astma-Allergi Danmark. Danish association for asthma and Allergi. Available: https://astma.astma-allergi.dk/boern/tal [Accessed 6 May 2019].

2 de Benedictis FM, Bush A. Infantile wheeze: rethinking dogma. Arch Dis Child 2017:102:371-5.

3 Claahsen-van der Grinten HL, Stikkelbroeck NMML, Otten BJ, et al. Congenital adrenal hyperplasia--pharmacologic interventions from the prenatal phase to adulthood. Pharmacol Ther 2011;132:1-14.

4 Danish Medicines Agency. Summary of Product Characteristics. Prednisolon "DAK, 2017. Available: www.produktresume.dk

5 Merke DP, Cho D, Calis KA, et al. Hydrocortisone suspension and hydrocortisone tablets are not bioequivalent in the treatment of children with congenital adrenal hyperplasia. J Clin Endocrinol Metab 2001;86:441-5.

6 Mulla H, Hussain N, Tanna S, et al. Assessment of liquid captopril formulations used in children. Arch Dis Child 2011;96:293-6.

7 Kearns GL, Abdel-Rahman SM, Alander SW, et al. Developmental pharmacology--drug disposition, action, and therapy in infants and children. N Engl J Med 2003;349:1157-67.

8 European Medicines Agency. Guideline on the investigation of bioequivalence. Available: http://www.ema.europa.eu/docs/en GB/ document_library/Scientific_guideline/2010/01/WC500070039.pdf [Accessed 30 Jun 2017].

9 Francisco GE, Honigberg IL, Stewart JT, et al. In vitro and in vivo bioequivalence of commercial prednisone tablets. Biopharm Drug Dispos 1984:5:335-44.

10 Georgitis JW, Flesher KA, Szefler SJ. Bioavailability assessment of a liquid prednisone preparation. J Allergy Clin Immunol 1982;70:243-7.

11 Teeninga N, Guan Z, Freijer J, et al. Monitoring prednisolone and prednisone in saliva: a population pharmacokinetic approach in healthy volunteers. Ther Drug Monit 2013;35:485-92.

12 Teeninga N, Guan Z, Stevens J, et al. Population pharmacokinetics of prednisolone in relation to clinical outcome in children with nephrotic syndrome. Ther Drug Monit 2016;38:534-45.

13 Mistry P, Batchelor H. Evidence of acceptability of oral paediatric medicines: a review. J Pharm Pharmacol 2017;69:361-76.

14 Lucas-Bouwman ME, Roorda RJ, Jansman FG, et al. Crushed prednisolone tablets or oral solution for acute asthma? Arch Dis Child 2001:84:347-8.

15 Aljebab F, Alanazi M, Choonara I, et al. Observational study on the palatability and tolerability of oral prednisolone and oral dexamethasone in children in Saudi Arabia and the UK. Arch Dis Child 2018;103:83-8.

16 Ethical considerations for clinical trials Om medicinal products conducted with minors. Available: http://ec.europa.eu/health//sites/ 
health/files/files/clinicaltrials/2016_06_pc_guidelines/gl_1_consult. pdf [Accessed 6 Apr 2017].

17 Europese Commissie. Eu guidelines to good manufacturing practice, Annex 13, investigational medicinal products. Available: https://ec. europa.eu/health/sites/health/files/files/eudralex/vol-4/2009_06_ annex13.pdf [Accessed 1 May 2018].

18 Rani S, Pargal A. Bioequivalence: an overview of statistical concepts 2004;36:209-16.
19 Aljebab F, Choonara I, Conroy S. Systematic review of the toxicity of short-course oral corticosteroids in children. Arch Dis Child 2016;101:365-70.

20 Rose JQ, Nickelsen JA, Ellis EF, et al. Prednisolone disposition in steroid-dependent asthmatic children. J Allergy Clin Immunol 1981;67:188-93.

21 Bartoszek M, Brenner AM, Szefler SJ. Prednisolone and methylprednisolone kinetics in children receiving anticonvulsant therapy. Clin Pharmacol Ther 1987;42:424-32. 